\title{
El efecto del nitrato de potasio, del ácido giberélico y del ácido indolacético sobre la germinación de semillas de agraz (Vaccinium meridionale Swartz)
}

\section{Effect of potassium nitrate, gibberellic acid, and indoleacetic acid on seed germination of Andean blueberry (Vaccinium meridionale Swartz)}

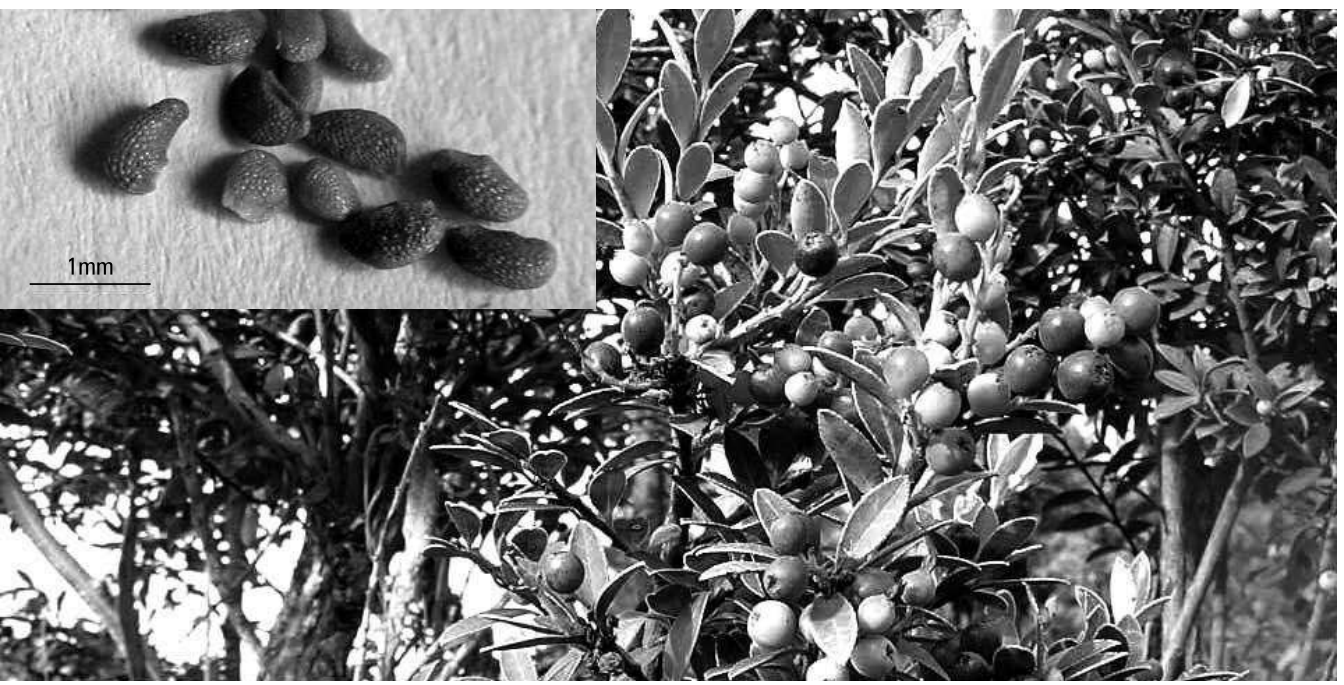

Semillas y planta de agraz en fructificación (Guachetá, Cundinamarca, Colombia).

Fotos: S.V. Magnitskiy

\section{RESUMEN}

El agraz es una planta silvestre de Suramérica con alto potencial entre las frutas comestibles para el mercado nacional e internacional. El presente estudio tuvo como objetivo evaluar los efectos del nitrato de potasio, el ácido giberélico y el ácido indolacético sobre la germinación de semillas de esta especie. Se demostró que las semillas almacenadas durante un mes dentro del fruto o extraídas presentaron 70 o $82 \%$ de viabilidad, respectivamente, 29 o 38\% de germinación a 35 días. La imbibición de las semillas en las soluciones con 200-500 $\mathrm{mg} \cdot \mathrm{L}^{-1}$ de nitrato de potasio o ácido giberélico aumentó el porcentaje de germinación en 32\% (semillas extraídas almacenadas), y 31 o $38 \%$ (semillas almacenadas en frutas), respectivamente, comparado con el testigo (sin aplicación), mientras que los tratamientos con el ácido indolacético no tuvieron un efecto significativo sobre la germinación. Los resultados mostraron que las semillas de agraz se caracterizan por poseer cierto mecanismo de latencia.

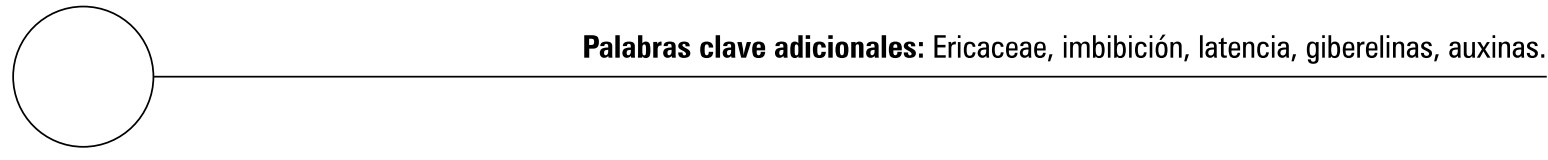

ProfesorAsistente,FacultaddeAgronomía,Universidad NacionaldeColombia,Bogotá(Colombia).svmagnitskiy@unal.edu.co ProfesorAsociado,FacultaddeAgronomía,UniversidadNacionaldeColombia,Bogotá(Colombia).galigarretom@unal.edu.co 


\section{ABSTRACT}

The Andean blueberry is a South American wild plant with a high exportation potential among all eatable fruits for national and international markets. The current research was undertaken to evaluate the effects of potassium nitrate, gibberellic acid, and indoleacetic acid on the seed germination of this specie. Results showed that seeds stored inside fruits for a month or extracted from fruits presented 70 or $82 \%$ viability, respectively, and 29 or $38 \%$ germination at day 35 . The imbibition of seeds in the solutions of $200-500 \mathrm{mg} \cdot \mathrm{L}^{-1}$ potassium nitrate or gibberellic acid, increased the germination percentage on $32 \%$ (extracted seed storage), and 31 or $38 \%$ (seeds stored inside fruits), respectively, as compared with control treatment, whereas the treatments with indoleacetic acid did not have a significant effect on the germination. The results indicated that seeds of Andean blueberry are characterized by some mechanisms of seed dormancy.

Additional key words: Ericaceae, imbibition, dormancy, gibberellins, auxins.

El agraz (Vaccinium meridionale Swartz, Ericaceae) es una planta silvestre nativa de las regiones andinas de Suramérica con alto potencial como frutas comestibles para el mercado nacional e internacional (Vallejo, 2000; Ligarreto et al., 2006; Salinas y Betancur, 2007). Su propagación asexual, por estacas, ha presentado varios problemas incluyendo el bajo porcentaje de enraizamiento y la larga duración del mismo (Medina y Lobo, 2004; Castrillon et al., 2007). Las semillas podrían servir como fuente de material para propagación de esta especie si se estableciera un protocolo adecuado de germinación. Por otro lado, las semillas de agraz, como la mayoría de especies Vaccinium (Jamieson y Nickerson, 2003), tienen una germinación errática y bajo porcentaje de germinación en condiciones controladas (Valencia y Ramírez, 1993). Se supone que las semillas de agraz son fotoblásticas positivas (Medina y Lobo, 2004); además, el porcentaje de germinación puede variar en depen- dencia con el tamaño de las semillas (Valencia y Ramírez, 1993), lo que implica la presencia de algún tipo de latencia. Teniendo en cuenta que el nitrato de potasio y el ácido giberélico pueden superar parcialmente la latencia en semillas, se hace importante evaluar su efecto sobre la germinación de las semillas de agraz. El uso de auxinas para romper la latencia en semillas con los fines prácticos ha sido poco estudiado, y varios autores reportan que estos reguladores de crecimiento tienen un efecto nulo (Copeland y McDonald, 2004), así como positivo (Ramakrishna y Ramakrishna, 2006) sobre la inducción de la germinación y el rompimiento de la latencia de semillas. El presente estudio tuvo como objetivo la evaluación del efecto del nitrato de potasio, el ácido giberélico y el ácido indolacético sobre la germinación de las semillas de agraz, con el fin de establecer los agentes promisorios para desarrollar el protocolo de germinación. 


\section{MATERIALES Y MÉTODOS}

Las frutas de agraz fueron colectadas en marzo de 2007 en el municipio de Guachetá (Cundinamarca, Colombia) y trasladadas al laboratorio de fisiología de cultivos, Universidad Nacional de Colombia, sede Bogotá, donde las semillas fueron extraídas manualmente, se secaron a $22^{\circ} \mathrm{C}$ durante $48 \mathrm{~h}$ y almacenadas durante 30 días a $5^{\circ} \mathrm{C}$. El otro grupo de semillas se almacenó en las mismas condiciones y se extrajo de las frutas inmediatamente antes de los tratamientos pregerminativos. Para los experimentos fueron utilizadas semillas de todos los tamaños.

Se aplicaron las recomendaciones del ISTA (1996) y del IBPGR (1985) para administrar las pruebas de viabilidad y germinación, teniendo en cuenta que las plantas del género Vaccinium no aparecen en la lista de las plantas de las reglas publicadas por ISTA. La prueba de viabilidad de las semillas se hizo después de sacar las semillas del cuarto de almacenamiento, se utilizaron por tratamiento 100 semillas en cuatro repeticiones y se expusieron a una solución de $0,5 \%$ de cloruro de 2, 3, 5-trifenil-tetrazolio durante $2 \mathrm{~h}$, a $38^{\circ} \mathrm{C}$. Posteriormente se procedió al corte de las semillas. Durante la prueba de germinación, 100 semillas en cuatro repeticiones se colocaron en cajas de Petri sobre papel Wattman en el germinador a $25^{\circ} \mathrm{C}$ a $8 \mathrm{~h}$ de luz con el tratamiento pregerminativo: control (agua destilada), 100, 200 ó $500 \mathrm{mg} \cdot \mathrm{L}^{-1}$ soluciones de $\mathrm{KNO}_{3}$, ácido giberélico $\left(\mathrm{GA}_{3}\right)$, o ácido indolacético (AIA). La primera y la segunda lecturas del conteo del porcentaje de germinación fueron administradas a los 22 y 35 días después del inicio de la germinación, respectivamente. Cada lectura estuvo acompañada de una clasificación de las plántulas en normales y anormales (Valencia y Ramírez, 1993; ISTA, 1996). Se realizó un análisis de varianza y la prueba de separación de promedios de Tukey con un nivel de significancia del 5\% para cada variable mediante el paquete estadístico SAS.

\section{RESULTADOS Y DISCUSIÓN}

La prueba de viabilidad antes de germinar las semillas demostró $70 \%$ y $82 \%$ de viabilidad de semillas cuando fueron almacenadas en el fruto o extraídas del fruto, respectivamente. Estos resultados concuerdan con datos ya reportados (Valencia y Ramírez, 1993), lo cual sugiere que el almacenamiento de semillas de Vaccinium sp. dentro de un fruto puede disminuir su viabilidad y por eso no es recomendable (Aalders y Hall, 1975). La prueba de tetrazolio representó un gran desafío para el estudio de viabilidad debido al diminuto tamaño de las semillas de agraz, por lo que se puede sugerir el uso de otros métodos, como la calorimetría (Magnitskiy et al., 2005), la cual ofrece ventajas en términos de rapidez y claridad de los resultados. Los resultados de la prueba de germinación se muestran en la tabla 1.

La germinación de las semillas de agraz fue lenta y alcanzó al final del experimento 29 y $38 \%$ en los controles para las semillas almacenadas en las frutas o extraídas, respectivamente (tabla 1). El porcentaje de plántulas normales al terminar la prueba de germinación fue de $96 \%$ para todos los tratamientos (datos no presentados). La imbibición de las semillas en las soluciones con 500 $\mathrm{mg} \cdot \mathrm{L}^{-1}$ del nitrato de potasio o ácido giberélico aumentó el porcentaje de germinación al día 35 en 32 y $32 \%$ (semillas almacenadas extraídas), respectivamente, o 31 y $38 \%$ (semillas almacenadas en frutas), respectivamente, comparable con los controles, mientras que los tratamientos con el ácido indolacético no tuvieron un efecto significativo sobre este parámetro (tabla 1).

Los porcentajes de viabilidad mayores que los de germinación en semillas sin tratamiento (controles) sugieren algún tipo de latencia, aunque cabe anotar que no se sabe si las condiciones de germinación fueron óptimas en este experimento, porque no están establecidas para esta especie. 
Tabla 1. Germinación de semillas de agraz (Vaccinium meridionale Swartz), imbibidas en soluciones de nitrato de potasio, ácido giberélico y ácido indolacético, extraídas de frutas $(E)$ o en frutas $(F)$, previamente almacenadas durante 30 días a $5^{\circ} \mathrm{C}$.

\begin{tabular}{|c|c|c|c|c|c|}
\hline & & \multicolumn{4}{|c|}{ Germinación (\%) } \\
\hline & & \multicolumn{2}{|c|}{22 días } & \multicolumn{2}{|c|}{35 días } \\
\hline & & E & $\mathrm{F}$ & E & $\mathrm{F}$ \\
\hline \multicolumn{2}{|c|}{$\mathrm{H}_{2} \mathrm{O}$ (control) } & $12 \mathrm{a}$ & $10 a$ & $38 a$ & $29 a$ \\
\hline \multirow{3}{*}{$\begin{array}{l}\mathrm{KNO}_{3} \\
\mathrm{mg} \cdot \mathrm{L}^{-1}\end{array}$} & 100 & $14 a$ & $10 a$ & $46 a b$ & $30 a$ \\
\hline & 200 & $14 a$ & $12 a$ & $48 b$ & $36 a b$ \\
\hline & 500 & $16 a b$ & $12 a$ & $50 \mathrm{~b}$ & $38 a b$ \\
\hline \multirow{3}{*}{$\begin{array}{l}\mathrm{GA}_{3^{\prime}} \\
\mathrm{mg} \cdot \mathrm{L}^{-1}\end{array}$} & 100 & $14 a$ & $14 a b$ & $44 a b$ & $32 a$ \\
\hline & 200 & $15 a$ & $12 a$ & $47 a b$ & $36 a b$ \\
\hline & 500 & $16 a b$ & $14 a b$ & $50 \mathrm{~b}$ & $40 b$ \\
\hline \multirow{3}{*}{$\begin{array}{l}\text { AIA, } \\
\mathrm{mg} \cdot \mathrm{L}^{-1}\end{array}$} & 100 & $12 a$ & $10 \mathrm{a}$ & $39 a$ & $32 a$ \\
\hline & 200 & $12 a$ & $12 \mathrm{a}$ & $38 a$ & $30 a$ \\
\hline & 500 & $12 \mathrm{a}$ & $11 \mathrm{a}$ & $38 a$ & $32 a$ \\
\hline
\end{tabular}

Promedios seguidos con la misma letra no presentan diferencia significativa según la prueba de Tukey $(P>0,05)$.

La existencia de latencia en semillas de agraz se justifica con más claridad observando el efecto de los tratamientos con nitrato de potasio y acido giberélico, los cuales aumentaron el porcentaje de germinación en relación con el que se encontró en los controles. El efecto observado con nitrato de potasio y ácido giberélico es bien conocido en otras especies (Devlin y Karczmarczyk, 1977; Giba et al., 1995) y se puede explicar porque suplen los requerimientos del sistema de fitocromo en semillas fotobásticas (Murdoch y Ellis, 2000), lo que puede implicar que las semillas de agraz se caracterizan por ciertos mecanismos de latencia fisiológica (Copeland y McDonald, 2004). Además, el ácido giberélico es conocido por disminuir los requerimientos de semillas para la estratificación caliente (McDonald, 2002), tal como en el caso de latencia morfológica relacionada con el embrión inmaduro, o estratificación fría, la cual a menudo es necesaria para inducir la germinación en las semillas de Vaccinium sp. de clima frío (IPBGR, 1985).

Se supone que una gran variedad de sustancias químicas típicas del piso de bosque promueven o inhiben la germinación de semillas en especies silvestres, como el agraz, que es un terreno grande por explorar mediante la investigación (Hilhorst y Karssen, 2000). Si cierta cantidad de plantas de agraz en su hábitat natural se propagaran sexualmente, sería útil proponer el estudio del efecto de las sustancias químicas o factores típicos del microambiente del banco de semillas para establecer las condiciones óptimas de germinación de semillas de agraz y eliminar efectos de latencia. Para especificar el tipo de latencia en semillas de agraz sería necesario estudiar la presencia de características fotoblásticas de las mismas. Con fines prácticos, se recomienda la utilización de soluciones de nitrato de potasio y $\mathrm{GA}_{3}$ en concentraciones $200-500 \mathrm{mg} \cdot \mathrm{L}^{-1}$ para promover la germinación de las semillas de esta especie bajo condiciones controladas.

\section{AGRADECIMIENTOS}

Los autores agradecen a COLCIENCIAS por la financiación del trabajo de investigación como parte integral del proyecto "Zonificación de especies de agraz y una aproximación a su manejo agronómico" realizado por la Facultad de Agronomía de la Universidad Nacional de Colombia, sede Bogotá. 


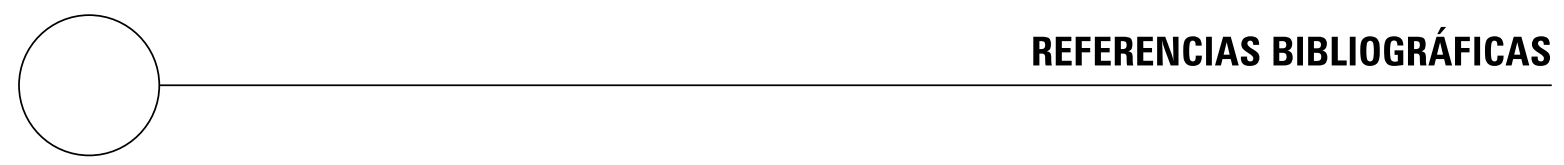

Aalders, L.E. y I.V. Hall. 1975. Germination of lowbush blueberry seeds stored dry and in fruit at different temperatures. HortScience 10, 525-526

Castrillon, J.C.; E. Carvajal; G.A. Ligarreto; S. Magnitskiy y J.D. Muñoz. 2007. Evaluación del efecto de auxinas en diferentes concentraciones sobre el enraizamiento de agraz (Vaccinium meridionale Swartz). pp. 116. En: Memorias Segundo Congreso Colombiano de Horticultura, Bogotá. 260 p.

Copeland, L.O. y M.B. McDonald. 2004. Principles of seed science and technology. 4th edition. Kluwer Academic Publishers. 467 p.

Devlin, R.M. y S.J. Karczmarczyk. 1977. Influence of light and growth regulators on cranberry seed dormancy. J. Hort. Sci. 52, 283-288.

Giba, Z.; D. Grubisic y R. Konjevic, 1995. The involvement of phytochrome in light-induced germination of blueberry (Vaccinium myrtillus L.) seeds. Seed Sci. Technol. 23, 11-19.

IBPGR. 1985. Ericaceae. Chapter 36. En: Handbook of seed technology for genebanks - Volume II. Compendium of specific germination information and test recommendations. International Board for Plant $\mathrm{Ge}$ netic Resources, Roma. 200 p.

ISTA. 1996. Internacional rules for seed testing. Internacional Seed Testing Association, Zürich. 335 p.

Jamieson, A.R. y N.L. Nickerson. 2003. Field performance of the lowbush blueberry propagated by seed, stem cuttings and micropropagation. Acta Hort. 626, 423-428.

Hilhorst, H.W.M. y C.M. Karssen. 2000. Effects of chemical environment on seed germination. pp. 293-309. En: Fenner, M. (ed.). Seeds: the ecology of regeneration in plants communities. CAB International.

Ligarreto, G.A.; C.I. Medina; L.B. Arias; L.J. Martínez; H. Corredor, J.D. Muñoz y P. Patiño. 2006. Zonificación de las especies de agraz (Vaccinium spp.) y una aproximación de su manejo agronómico como cultivos promisorios para la zona alto andina colombiana. Segundo informe de actividades. Universidad Nacional de Colombia, Facultad Agronomía, Bogotá. 26 p.

Magnitskiy, S.V.; C.C. Pasian; D.W. Burger; M.A. Bennett y J.D. Metzger. 2005. Viability, metabolic heat, and respiration rates of paclobutrazol treated verbena and marigold seeds. Seed Technol. 27(2), 223-229.

McDonald, M.B. 2002. A philosophy of dormancy testing in native species. Seed Technol. 24(1), 26-35.

Medina, C.I. y M.A. Lobo, 2004. Colecta, conservación y caracterización de diversas poblaciones de Vaccinium meridionale (mortiño), presentes en los bosques altoandinos de la jurisdicción de Corantioquia para promover su utilización sostenible. Informe final técnico del proyecto. Corpoica, Rionegro. 32 p.

Murdoch, A.J. y R.H. Ellis. 2000. Fenner, M. (ed.). Dormancy, viability and longevity. pp. 183-214. En: Ferner, M. (ed.). Seeds: the ecology of regeneration in plants communities. $\mathrm{CAB}$ Internacional.

Ramakrishna, V. y P.R. Ramakrishna. 2006. Effect of in vivo administered plant growth hormones on the development of amylase and protease during germination of indian bean (Dolichos lablab L. var. lignosus). Acta Physiol. Plant. 28, 245-260.

Salinas, R.N. y J. Betancur. 2007. Novedades taxonómicas de las Ericáceas del Suroccidente de Colombia. Caldasia 29(1), 51-58.

Valencia, M.L.C. de y F. Ramírez. 1993. Notas sobre la morfología, anatomía y germinación del agraz (Vaccinium meridionale Swartz). Agron. Colomb. 10(2), 151-159.

Vallejo, D.A. 2000. Fomento al mortiño (Vaccinium meridionale) como especie promisoria del Parque Regional Arví. Corantioquia. $20 \mathrm{p}$. 